\title{
NMR Spectral correlations in some Tröger's bases
}

\author{
G. Thirunarayanan*, M. Suresh \\ Department of Chemistry, Annamalai University, Annamalainagar 608002, India \\ E-mail address: drgtnarayanan@gmail.com
}

\begin{abstract}
Some Tröger's bases have been synthesised from substituted anilines and paraformaldehyde in presence of Lewis acid catalyst such as anhydrous $\mathrm{AlCl}_{3}$, through electrophilic substitution reaction. The purities of these Tröger's bases have been checked by their physical constants and spectroscopic data published earlier in the literature. The NMR chemical shift $(\delta, \mathrm{ppm})$ of methylene protons and carbon were assigned. The assigned methylene protons and carbon chemical shifts $(\delta, \mathrm{ppm})$ of synthesised Tröger's bases have been correlated with Hammett substituent constants, $\mathrm{F}$ and $\mathrm{R}$ parameters using single and multi-regression analyses. From the results of statistical analyses, the effects of substituent on methylene protons and carbon were discussed.
\end{abstract}

Keywords: Tröger's bases; NMR spectra; Hammett correlations; Substituent effects

\section{INTRODUCTION}

Tröger's base (Fig. 1), is a molecule containing chiral nitrogen centres exists in two eneatiomeric form. Due to its rigid and concave shape, it has attracted an intense research in the last several decades [1]. Its chiral nature was first identified by Prelog and Wieland in 1944 [2]. Many Tröger's bases of the type whether substituted or condensed aromatic rings have been reported as $8 \mathrm{H}, 16 \mathrm{H}-7,15$-methanodinaphtho[2,1-b][2',1'-f][1,5]-diazocine, was synthesized by Tálas et al., [3]. Organic chemists synthesized various kinds of Tröger's base derivatives from $\mathrm{C}$ - amino heterocycles $\left[4,5\right.$, naphthalimide flurophore[ 6 , rigid $\mathrm{C}_{2}$-symmetric crown ether [7], Chiral primary bis-ammonium salts [8], rearrangement of pyrazolines [9] mercaptanes [1], halogenation [10] and N-methyl pyrrole units [11,12]. The mechanism of formation of Tröger's base derivatives were investigated Abella et.al., [13] through AEI Mass spectral data. Chiral recognition by phenomena [14,15] was important for various fields likeracemic mixture resolution, determination of enantiomeric purity of chiral compounds and the selectivity of catalysts. Periasamy and Sathishkumar investigated the chiral recognition of carboxylic acids in the Tröger's base derivatives [14,15]. Several catalysts were employed for the synthesis of enantioselective rigid Tröger's base derivatives such as $\mathrm{TiCl}_{4}, \mathrm{AlCl}_{3}, \mathrm{SnCl}_{4}, \mathrm{ZnCl}_{2}, \mathrm{ZrCl}_{4}$ [14], TFFA [16], $\mathrm{Na}_{2} \mathrm{CO}_{3}$ [17]. Didier and Sergeyev synthesized a few symmetrical amino and amino ethyl Tröger's base derivatives via $\mathrm{Pd}$ catalyzed C-C and C-N bond formation [18]. 


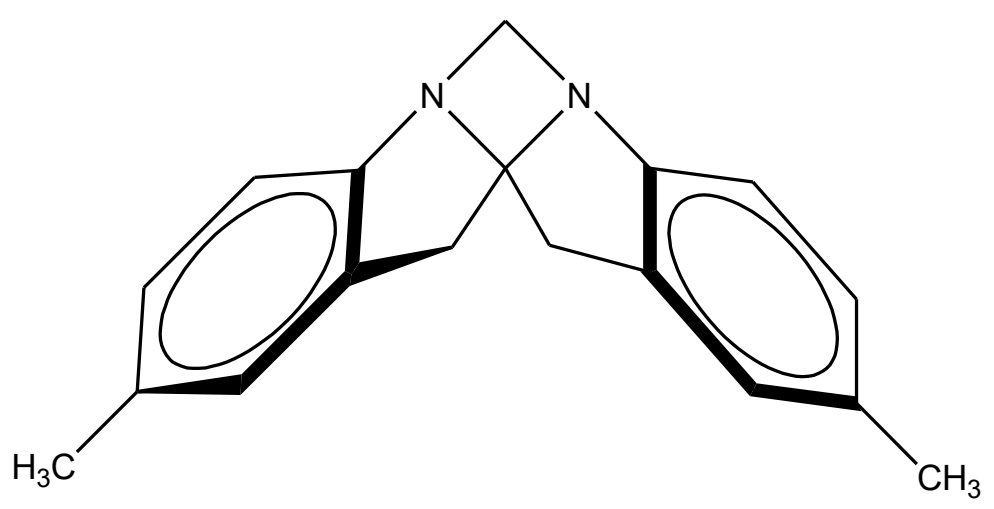

Fig. 1. General structure of Tröger's base.

Tröger's base derivatives are also used as catalysts for organic synthesis-construction of new supramolecular hosts [19,20], complexation [21], diastereoselective self-assembly of double standard helicates [21,23], molecular tweezers [24,25], scaffolds [26,27] and HPLC chromophoric solid phase supporters [28-30]. The synthesis of pure optically active Ru(II) complexes with a chiral Tröger's base ligands and their interaction towards DNA was investigated by Classens et al., [31]. DFT phenomena and NMR spectroscopy data were used for investigation of the various properties of Tröger's base derivatives [24]. Spectroscopic data is useful for prediction of ground state equilibration of molecules [32]. Generally compounds including Tröger's bases which are having hetero atom like $\mathrm{O}, \mathrm{S}, \mathrm{N}$ and notified functional group such as carbonyl, alkene, alkynes, halogens possess biological activities [33]. Herein, the authors wishes to report the synthesis of a series of enantioselective Tröger's base derivatives and studied the effects of substituent on methylene protons and carbons from their NMR chemical shifts(ppm) using single and multi-linear regression analysis.

\section{EXPERIMENTAL}

\section{1. Materials and methods}

All chemicals used were purchased from Sigma-Aldrich and E-Merck chemical companies. Melting points of all Tröger's bases were determined in open glass capillaries on Mettler FP51 melting point apparatus and are uncorrected. Infrared spectra (KBr, 4000-400 $\mathrm{cm}^{-1}$ ) were recorded on AVATAR-300 Fourier transform spectrophotometer. The NMR spectra are recorded in INSTRUM AV500 NMR spectrometer, operating at $500 \mathrm{MHz}$ for ${ }^{1} \mathrm{H}$ spectra and $125.46 \mathrm{MHz}$ for ${ }^{13} \mathrm{C}$ spectra in $\mathrm{CDCl}_{3}$ solvent using TMS as internal standard. Electron impact $(\mathrm{EI})(70 \mathrm{eV})$ and $\mathrm{FAB}^{+}$mass spectra were recorded using VARIAN 500 mass spectrometer.

\section{2. General procedure for the synthesis of substituted Tröger's bases [33]}

To an appropriate molar mass of substituted anilines $(10 \mathrm{mmol})$ and paraformaldehyde (20 mmol) in $\mathrm{CH}_{2} \mathrm{Cl}_{2}(30 \mathrm{~mL})$, anhydrous $\mathrm{AlCl}_{3}(1.36 \mathrm{~g}, 10 \mathrm{mmol})$ was added under $\mathrm{N}_{2}$ atmosphere. The reaction mixture was stirred for $12 \mathrm{~h}$ at $25{ }^{\circ} \mathrm{C}$ (Scheme 1) and quenched with cold water $(10 \mathrm{~mL})$. The reaction mixture was extracted with $\mathrm{CH}_{2} \mathrm{Cl}_{2}(20 \mathrm{~mL})$ and the combined organic extracts were successively washed with water, brine and dried over 
anhydrous $\mathrm{Na}_{2} \mathrm{SO}_{4}$. After removal of the solvent, the residue was subjected to chromatography on alumina (basic) column using $10 \%$ ethyl acetate in hexane to elute the desired Tröger's base analogs [14,15,33]. The analytical and Mass and NMR spectroscopic data are presented in Table 1.<smiles>[R]c1cc(N)c([R])c([R])c1[R]</smiles>

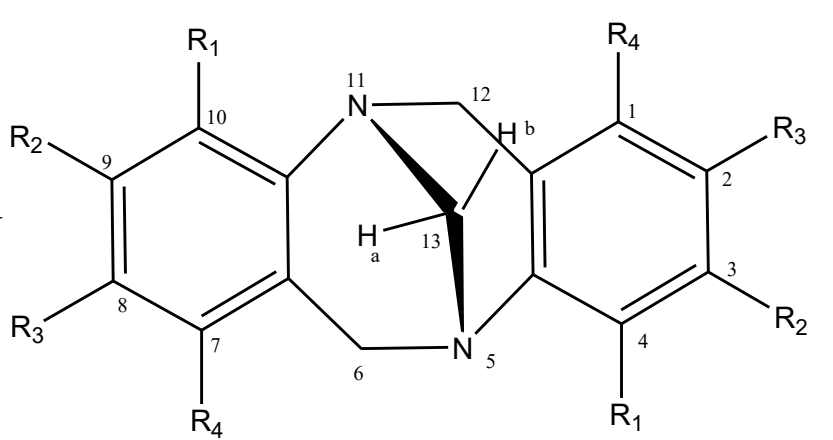

\begin{tabular}{|c|c|c|c|c|}
\hline Entry & $\mathbf{R}_{\mathbf{1}}$ & $\mathbf{R}_{\mathbf{2}}$ & $\mathbf{R}_{\mathbf{3}}$ & $\mathbf{R}_{\mathbf{4}}$ \\
\hline $\mathbf{1}$ & $\mathrm{H}$ & $\mathrm{H}$ & $\mathrm{H}$ & $\mathrm{H}$ \\
\hline $\mathbf{2}$ & $\mathrm{H}$ & $\mathrm{H}$ & $\mathrm{NH}_{2}$ & $\mathrm{H}$ \\
\hline $\mathbf{3}$ & $\mathrm{Br}$ & $\mathrm{H}$ & $\mathrm{H}$ & $\mathrm{H}$ \\
\hline $\mathbf{4}$ & $\mathrm{H}$ & $\mathrm{Br}$ & $\mathrm{H}$ & $\mathrm{H}$ \\
\hline $\mathbf{5}$ & $\mathrm{H}$ & $\mathrm{H}$ & $\mathrm{Br}$ & $\mathrm{H}$ \\
\hline $\mathbf{6}$ & $\mathrm{Cl}$ & $\mathrm{H}$ & $\mathrm{H}$ & $\mathrm{H}$ \\
\hline $\mathbf{7}$ & $\mathrm{H}$ & $\mathrm{Cl}$ & $\mathrm{H}$ & $\mathrm{H}$ \\
\hline $\mathbf{8}$ & $\mathrm{H}$ & $\mathrm{H}$ & $\mathrm{Cl}$ & $\mathrm{H}$ \\
\hline $\mathbf{9}$ & $\mathrm{H}$ & $\mathrm{H}$ & $\mathrm{CN}$ & $\mathrm{H}$ \\
\hline $\mathbf{1 0}$ & $\mathrm{F}$ & $\mathrm{H}$ & $\mathrm{H}$ & $\mathrm{H}$ \\
\hline $\mathbf{1 1}$ & $\mathrm{H}$ & $\mathrm{F}$ & $\mathrm{H}$ & $\mathrm{H}$ \\
\hline $\mathbf{1 2}$ & $\mathrm{H}$ & $\mathrm{H}$ & $\mathrm{F}$ & $\mathrm{H}$ \\
\hline $\mathbf{1 3}$ & $\mathrm{H}$ & $\mathrm{H}$ & $\mathrm{OC}_{2} \mathrm{H}_{5}$ & $\mathrm{H}$ \\
\hline $\mathbf{1 4}$ & $\mathrm{H}$ & $\mathrm{H}$ & $\mathrm{COOC}_{2} \mathrm{H}_{5}$ & $\mathrm{H}$ \\
\hline $\mathbf{1 5}$ & $\mathrm{H}$ & $\mathrm{H}$ & $\mathrm{OCH}_{3}$ & $\mathrm{H}$ \\
\hline $\mathbf{1 6}$ & $\mathrm{H}$ & $\mathrm{H}$ & $\mathrm{CH}_{3}$ & $\mathrm{H}$ \\
\hline $\mathbf{1 7}$ & $\mathrm{H}$ & $\mathrm{H}$ & $\mathrm{NO}_{2}$ & $\mathrm{H}$ \\
\hline $\mathbf{1 8}$ & $\mathrm{H}$ & $\mathrm{H}$ & $\mathrm{CF}_{3}$ & $\mathrm{H}$ \\
\hline
\end{tabular}

Scheme 1. Synthesis of Tröger's bases 


\section{RESULTS AND DISCUSSION}

\section{1. ${ }^{1}$ H NMR spectral study}

In the present study, the compounds chosen for evaluating the effects of substituents on methylene unit in Tröger's bases is shown in Scheme 1. The NMR spectra of synthesised Tröger's bases were recorded in $\mathrm{CDCl}_{3}$ solvent using TMS as internal standard. The Chemical shifts $(\delta, \mathrm{ppm})$ of methylene protons and carbons of synthesised Tröger's bases were presented in Table 1 . These chemical shifts $(\delta, \mathrm{ppm})$ have been correlated with Hammett substituent constants, $\mathrm{F}$ and $\mathrm{R}$ parameters using single and multi-linear regression analysis $[32,34-44]$. In this correlation the Hammett equation was taken in the form as

$$
\delta=\rho \sigma+\delta_{0}
$$

where $\delta_{\mathrm{o}}$ is the frequency for the parent member of the series.

The results of statistical analyses of correlation of $\delta \mathrm{Ha}$ and $\mathrm{Hb}$ chemical shifts (ppm) of Tröger's bases with Hammett substituent constants, F and R parameters were presented in Table 2. From Table 2, the correlation of $\delta \mathrm{Ha}$ chemical shifts (ppm) values correlated satisfactorily with Hammett substituent constants and $\mathrm{F}$ parameters individually except $\mathrm{NH}_{2}$, 4- $\mathrm{OCH}_{3}, 4-\mathrm{COOC}_{2} \mathrm{H}_{5}, 4-\mathrm{NO}_{2}, 4-\mathrm{CN}, 4-\mathrm{OC}_{2} \mathrm{H}_{5}$ and 4-F substituents. The resonance parameter was failed in correlation with these frequencies. This is due to the lone pair of nitrogen atom opposes the of effect of substituents on the methylene moiety and is associated with the resonance conjugative structure shown in Fig. 2. All correlations gave positive $\rho$ values except $\sigma_{R}$ constants and Resonance parameters.

Table 1. Physical constants, microanalysis mass (m/z) fragments and NMR chemical shifts $(\delta, \mathrm{ppm})$ of Tröger's bases.

\begin{tabular}{|c|c|c|c|c|c|c|c|}
\hline \multirow{2}{*}{ Entry } & \multirow{2}{*}{ M. F. } & \multirow{2}{*}{ M. W. } & \multirow{2}{*}{ M.p. $\left({ }^{\circ} \mathrm{C}\right)$} & \multirow{2}{*}{$\operatorname{Mass}(\mathrm{m} / \mathrm{z})$} & \multicolumn{2}{|c|}{${ }^{1} \mathrm{H}$ NMR } & \multirow{2}{*}{$\begin{array}{c}{ }^{13} \mathrm{C} \\
\mathrm{NMR}\end{array}$} \\
\hline & & & & & $\begin{array}{c}\mathrm{Ha} \\
(1 \mathrm{H}, d)\end{array}$ & $\begin{array}{c}\mathrm{Hb} \\
(1 \mathrm{H}, d)\end{array}$ & \\
\hline 1 & $\mathrm{C}_{15} \mathrm{H}_{14} \mathrm{~N}_{2}$ & 222 & $\begin{array}{c}131-132 \\
(130-131) \\
{[33]}\end{array}$ & $222\left[\mathrm{M}^{+}\right]$ & 4.065 & 4.117 & 66.721 \\
\hline 2 & $\mathrm{C}_{15} \mathrm{H}_{16} \mathrm{~N}_{4}$ & 252 & $\begin{array}{c}267-268 \\
(267-268) \\
{[33]} \\
\end{array}$ & $252\left[\mathrm{M}^{+}\right]$ & 4.601 & 4.760 & 66.632 \\
\hline 3 & $\mathrm{C}_{15} \mathrm{H}_{12} \mathrm{~N}_{2} \mathrm{Br}_{2}$ & 378 & $\begin{array}{c}162-163 \\
(162-163) \\
{[33]}\end{array}$ & $\begin{array}{c}378\left[\mathrm{M}^{+}\right], \\
380\left[\mathrm{M}^{2+}\right] \\
382\left[\mathrm{M}^{4+}\right]\end{array}$ & 4.617 & 4.692 & 66.817 \\
\hline 4 & $\mathrm{C}_{15} \mathrm{H}_{12} \mathrm{~N}_{2} \mathrm{Br}_{2}$ & 378 & $\begin{array}{c}203-204 \\
(203-204) \\
{[33]}\end{array}$ & $\begin{array}{c}378\left[\mathrm{M}^{+}\right] \\
380\left[\mathrm{M}^{2+}\right] \\
382\left[\mathrm{M}^{4+}\right]\end{array}$ & 4.671 & 4.690 & 66.714 \\
\hline 5 & $\mathrm{C}_{15} \mathrm{H}_{12} \mathrm{~N}_{2} \mathrm{Br}_{2}$ & 378 & $\begin{array}{c}163-164 \\
(163-164)\end{array}$ & $\begin{array}{c}378\left[\mathrm{M}^{+}\right] \\
380\left[\mathrm{M}^{2+}\right]\end{array}$ & 4.217 & 4.276 & 67.010 \\
\hline
\end{tabular}




\begin{tabular}{|c|c|c|c|c|c|c|c|}
\hline & & & [33] & $382\left[\mathrm{M}^{4+}\right]$ & & & \\
\hline 6 & $\mathrm{C}_{15} \mathrm{H}_{12} \mathrm{~N}_{2} \mathrm{Cl}_{2}$ & 290 & $\begin{array}{c}144-145 \\
(144-145) \\
{[33]}\end{array}$ & $\begin{array}{c}290\left[\mathrm{M}^{+}\right] \\
292\left[\mathrm{M}^{2+}\right] \\
294\left[\mathrm{M}^{4+}\right]\end{array}$ & 4.678 & 4.680 & 66.825 \\
\hline 7 & $\mathrm{C}_{15} \mathrm{H}_{12} \mathrm{~N}_{2} \mathrm{Cl}_{2}$ & 290 & $\begin{array}{c}192-193 \\
(192-193) \\
{[33]}\end{array}$ & $\begin{array}{c}290\left[\mathrm{M}^{+}\right], \\
292\left[\mathrm{M}^{2+}\right], \\
294\left[\mathrm{M}^{4+}\right]\end{array}$ & 4.680 & 4.687 & 66.619 \\
\hline 8 & $\mathrm{C}_{15} \mathrm{H}_{12} \mathrm{~N}_{2} \mathrm{Cl}_{2}$ & 290 & $\begin{array}{c}144-145 \\
(144-145) \\
{[33]}\end{array}$ & $\begin{array}{c}290\left[\mathrm{M}^{+}\right], \\
292\left[\mathrm{M}^{2+}\right] \\
294\left[\mathrm{M}^{4+}\right]\end{array}$ & 4.301 & 4.367 & 67.117 \\
\hline 9 & $\mathrm{C}_{17} \mathrm{H}_{12} \mathrm{~N}_{2}$ & 282 & $\begin{array}{c}249-250 \\
(249-250) \\
{[33]}\end{array}$ & $282\left[\mathrm{M}^{+}\right]$ & 4.021 & 4.137 & 66.715 \\
\hline 10 & $\mathrm{C}_{15} \mathrm{H}_{12} \mathrm{~N}_{2} \mathrm{~F}_{2}$ & 246 & $\begin{array}{c}123-124 \\
(123-124) \\
{[33]}\end{array}$ & $\begin{array}{c}246\left[\mathrm{M}^{+}\right], \\
248\left[\mathrm{M}^{2+}\right], \\
250\left[\mathrm{M}^{4+}\right]\end{array}$ & 4.687 & 4.717 & 66.901 \\
\hline 11 & $\mathrm{C}_{15} \mathrm{H}_{12} \mathrm{~N}_{2} \mathrm{~F}_{2}$ & 246 & $\begin{array}{c}140-141 \\
(140-141) \\
{[33]}\end{array}$ & $\begin{array}{c}246\left[\mathrm{M}^{+}\right] \\
248\left[\mathrm{M}^{2+}\right] \\
250\left[\mathrm{M}^{4+}\right]\end{array}$ & 4.601 & 4.651 & 66.517 \\
\hline 12 & $\mathrm{C}_{15} \mathrm{H}_{12} \mathrm{~N}_{2} \mathrm{~F}_{2}$ & 246 & $\begin{array}{c}117-118 \\
(117-118) \\
{[33]}\end{array}$ & $\begin{array}{c}246\left[\mathrm{M}^{+}\right], \\
248\left[\mathrm{M}^{2+}\right], \\
250\left[\mathrm{M}^{4+}\right]\end{array}$ & 4.311 & 4.357 & 66.781 \\
\hline 13 & $\mathrm{C}_{19} \mathrm{H}_{19} \mathrm{~N}_{2} \mathrm{O}_{2}$ & 310 & $\begin{array}{c}137-138 \\
(137-138) \\
{[33]}\end{array}$ & $310\left[\mathrm{M}^{+}\right]$ & 4.107 & 4.181 & 66.076 \\
\hline 14 & $\mathrm{C}_{21} \mathrm{H}_{22} \mathrm{~N}_{2} \mathrm{O}_{4}$ & 366 & $\begin{array}{c}125-126 \\
(125-126) \\
{[33]}\end{array}$ & $366\left[\mathrm{M}^{+}\right]$ & 4.055 & 4.103 & 66.472 \\
\hline 15 & $\mathrm{C}_{17} \mathrm{H}_{15} \mathrm{~N}_{2} \mathrm{O}_{2}$ & 282 & $\begin{array}{c}170-171 \\
(170-171) \\
{[33]} \\
\end{array}$ & $282\left[\mathrm{M}^{+}\right]$ & 4.015 & 4.017 & 66.027 \\
\hline 16 & $\mathrm{C}_{17} \mathrm{H}_{18} \mathrm{~N}_{2}$ & 250 & $\begin{array}{c}136-137 \\
(136-137) \\
{[33]}\end{array}$ & $250\left[\mathrm{M}^{+}\right]$ & 4.101 & 4.137 & 66.173 \\
\hline 17 & $\mathrm{C}_{15} \mathrm{H}_{12} \mathrm{~N}_{4} \mathrm{O}_{4}$ & 274 & $\begin{array}{c}256-257 \\
(256-257) \\
{[33]}\end{array}$ & $274\left[\mathrm{M}^{+}\right]$ & 4.192 & 4.271 & 67.217 \\
\hline 18 & $\mathrm{C}_{17} \mathrm{H}_{12} \mathrm{~N}_{2} \mathrm{~F}_{6}$ & 316 & $\begin{array}{c}129-130 \\
(129-130) \\
{[33]}\end{array}$ & $\begin{array}{c}316\left[\mathrm{M}^{+}\right], \\
318\left[\mathrm{M}^{2+}\right], \\
320\left[\mathrm{M}^{4+}\right]\end{array}$ & 4.176 & 4.192 & 66.817 \\
\hline
\end{tabular}


Table 2. Results of statistical analysis of NMR chemical shifts $(\delta, \mathrm{ppm})$ of methylene protons and carbon of Tröger's bases with Hammett substituent constants, $F$ and R parameters.

\begin{tabular}{|c|c|c|c|c|c|c|c|}
\hline Freq. & Constt. & $\mathbf{r}$ & I & $\rho$ & $\mathbf{S}$ & $\mathbf{n}$ & Correlated derivatives \\
\hline \multirow[t]{6}{*}{$\mathrm{Ha}$} & $\sigma$ & 0.933 & 4.264 & 0.226 & 0.26 & 13 & $\begin{array}{c}\mathrm{H}, 2-\mathrm{Br}, 3-\mathrm{Br}, 4-\mathrm{Br}, 2-\mathrm{Cl}, 3-\mathrm{Cl}, \\
\text { 4-Cl, 2-F, 3-F, 4-F, 4-OC } \mathrm{OH}_{5}, \\
\text { 4- } \mathrm{OCH}_{3}, 4-\mathrm{CH}_{3}\end{array}$ \\
\hline & $\sigma^{+}$ & 0.943 & 4.269 & 0.213 & 0.24 & 13 & $\begin{array}{c}\mathrm{H}, 2-\mathrm{Br}, 3-\mathrm{Br}, 4-\mathrm{Br}, 2-\mathrm{Cl}, 3-\mathrm{Cl}, \\
\text { 4-Cl, 2-F, 3-F, 4-F, 4-OC } 2 \mathrm{H}_{5}, \\
\text { 4- } \mathrm{OCH}_{3}, 4-\mathrm{CH}_{3}\end{array}$ \\
\hline & $\sigma_{\mathrm{I}}$ & 0.904 & 4.037 & 0.697 & 0.24 & 15 & 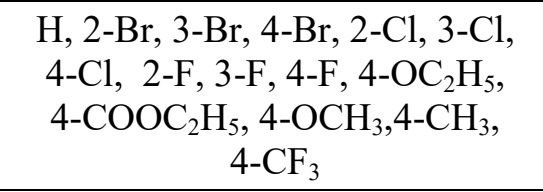 \\
\hline & $\sigma_{\mathrm{R}}$ & 0.902 & 4.243 & -0.305 & 0.24 & 14 & $\begin{array}{c}\mathrm{H}, 2-\mathrm{Br}, 3-\mathrm{Br}, 4-\mathrm{Br}, 2-\mathrm{Cl}, 3-\mathrm{Cl}, \\
\text { 4-Cl, 4-CN, 2-F, 3-F, } \\
\text { 4-COOC }{ }_{2} \mathrm{H}_{5}, 4-\mathrm{CH}_{3}, 4-\mathrm{NO}_{2}, 4-\mathrm{CF}_{3}\end{array}$ \\
\hline & $\mathrm{F}$ & 0.900 & 4.403 & 0.649 & 0.23 & 18 & $\begin{array}{c}\mathrm{H}, 4-\mathrm{NH}_{2}, 2-\mathrm{Br}, 3-\mathrm{Br}, 4-\mathrm{Br}, 2-\mathrm{Cl} \text {, } \\
\text { 3-Cl, 4-Cl, 4-CN, 2-F, 3-F, 4-F, } \\
\text { 4- } \mathrm{OC}_{2} \mathrm{H}_{5}, 4-\mathrm{COOC}_{2} \mathrm{H}_{5}, 4-\mathrm{OCH}_{3} \text {, } \\
\text { 4- } \mathrm{CH}_{3}, 4-\mathrm{NO}_{2}, 4-\mathrm{CF}_{3}\end{array}$ \\
\hline & $\mathrm{R}$ & 0.811 & 4.355 & -0.197 & 0.27 & 18 & $\begin{array}{c}\mathrm{H}, 4-\mathrm{NH}_{2}, 2-\mathrm{Br}, 3-\mathrm{Br}, 4-\mathrm{Br}, 2-\mathrm{Cl} \text {, } \\
\text { 3-Cl, 4-Cl, 4-CN, 2-F, 3-F, 4-F, } \\
\text { 4-OC }{ }_{2} \mathrm{H}_{5}, 4-\mathrm{COOC}_{2} \mathrm{H}_{5}, 4-\mathrm{OCH}_{3} \text {, } \\
\text { 4- } \mathrm{CH}_{3}, 4-\mathrm{NO}_{2}, 4-\mathrm{CF}_{3}\end{array}$ \\
\hline \multirow[t]{5}{*}{$\mathrm{Hb}$} & $\sigma$ & 0.926 & 4.316 & 0.191 & 0.25 & 13 & $\begin{array}{c}\mathrm{H}, 2-\mathrm{Br}, 3-\mathrm{Br}, 4-\mathrm{Br}, 2-\mathrm{Cl}, 3-\mathrm{Cl}, \\
\text { 4-Cl, 2-F, 3-F, 4-F, 4-OC } 2 \mathrm{H}_{5}, \\
\text { 4- } \mathrm{OCH}_{3}, 4-\mathrm{CH}_{3}\end{array}$ \\
\hline & $\sigma^{+}$ & 0.943 & 4.317 & 0.196 & 0.24 & 13 & $\begin{array}{c}\mathrm{H}, 2-\mathrm{Br}, 3-\mathrm{Br}, 4-\mathrm{Br}, 2-\mathrm{Cl}, 3-\mathrm{Cl} \text {, } \\
\text { 4-Cl, 2-F, 3-F, 4-F, 4-COOC } \mathrm{H}_{5} \text {, } \\
\text { 4- } \mathrm{OCH}_{3}, 4-\mathrm{CH}_{3}\end{array}$ \\
\hline & $\sigma_{\mathrm{I}}$ & 0.848 & 4.098 & 0.657 & 0.23 & 18 & $\begin{array}{c}\mathrm{H}, 4-\mathrm{NH}_{2}, 2-\mathrm{Br}, 3-\mathrm{Br}, 4-\mathrm{Br}, 2-\mathrm{Cl} \text {, } \\
\text { 3-Cl, 4-Cl, 4-CN, 2-F, 3-F, 4-F, } \\
\text { 4-OC } \mathrm{OC}_{2} \mathrm{H}_{5}, 4-\mathrm{COOC}_{2} \mathrm{H}_{5}, 4-\mathrm{OCH}_{3} \text {, } \\
\text { 4- } \mathrm{CH}_{3}, 4-\mathrm{NO}_{2}, 4-\mathrm{CF}_{3}\end{array}$ \\
\hline & $\sigma_{\mathrm{R}}$ & 0.831 & 4.283 & -0.330 & 0.25 & 18 & $\begin{array}{c}\mathrm{H}, 4-\mathrm{NH}_{2}, 2-\mathrm{Br}, 3-\mathrm{Br}, 4-\mathrm{Br}, 2-\mathrm{Cl} \text {, } \\
\text { 3-Cl, 4-Cl, 4-CN, 2-F, 3-F, 4-F, } \\
\text { 4-OC } \mathrm{OH}_{5}, 4-\mathrm{COOC}_{2} \mathrm{H}_{5}, 4-\mathrm{OCH}_{3} \text {, } \\
\text { 4- } \mathrm{CH}_{3}, 4-\mathrm{NO}_{2}, 4-\mathrm{CF}_{3}\end{array}$ \\
\hline & $\mathrm{F}$ & 0.905 & 4.103 & 0.620 & 0.22 & 18 & $\begin{array}{c}\mathrm{H}, 4-\mathrm{NH}_{2}, 2-\mathrm{Br}, 3-\mathrm{Br}, 4-\mathrm{Br}, 2-\mathrm{Cl} \text {, } \\
\text { 3-Cl, 4-Cl, 4-CN, 2-F, 3-F, 4-F, } \\
\text { 4- } \mathrm{OC}_{2} \mathrm{H}_{5}, 4-\mathrm{COOC}_{2} \mathrm{H}_{5}, 4-\mathrm{OCH}_{3} \text {, } \\
\text { 4- } \mathrm{CH}_{3}, 4-\mathrm{NO}_{2}, 4-\mathrm{CF}_{3}\end{array}$ \\
\hline
\end{tabular}




\begin{tabular}{|c|c|c|c|c|c|c|c|}
\hline & $\mathrm{R}$ & 0.824 & 4.294 & -0.223 & 0.25 & 18 & $\begin{array}{c}\mathrm{H}, 4-\mathrm{NH}_{2}, 2-\mathrm{Br}, 3-\mathrm{Br}, 4-\mathrm{Br}, 2-\mathrm{Cl} \text {, } \\
\text { 3-Cl, 4-Cl, 4-CN, 2-F, 3-F, 4-F, } \\
\text { 4- } \mathrm{OC}_{2} \mathrm{H}_{5}, 4-\mathrm{COOC}_{2} \mathrm{H}_{5}, 4-\mathrm{OCH}_{3} \text {, } \\
\text { 4- } \mathrm{CH}_{3}, 4-\mathrm{NO}_{2}, 4-\mathrm{CF}_{3}\end{array}$ \\
\hline \multirow[t]{6}{*}{$\mathrm{CH}_{2}$} & $\sigma$ & 0.964 & 66.519 & 0.632 & 0.38 & 15 & $\begin{array}{c}\mathrm{H}, 2-\mathrm{Br}, 3-\mathrm{Br}, 4-\mathrm{Br}, 2-\mathrm{Cl}, 3-\mathrm{Cl}, \\
\text { 4-Cl, 2-F, 3-F, 4-F, 4-OC } \mathrm{OH}_{5} \\
\text { 4- } \mathrm{COOC}_{2} \mathrm{H}_{5}, 4-\mathrm{CH}_{3}, 4-\mathrm{NO}_{2}, 4-\mathrm{CF}_{3}\end{array}$ \\
\hline & $\sigma^{+}$ & 0.905 & 66.581 & 0.322 & 0.31 & 17 & $\begin{array}{c}\mathrm{H}, 4-\mathrm{NH}_{2}, 2-\mathrm{Br}, 3-\mathrm{Br}, 4-\mathrm{Br}, 2-\mathrm{Cl}, \\
\text { 3-Cl, 4-Cl, 4-CN, 2-F, 3-F, 4-F, } \\
\text { 4-COOC } \mathrm{H}_{5}, 4-\mathrm{OCH}_{3}, 4-\mathrm{CH}_{3}, \\
\text { 4- } \mathrm{NO}_{2}, 4-\mathrm{CF}_{3}\end{array}$ \\
\hline & $\sigma_{\mathrm{I}}$ & 0.900 & 66.213 & 1.103 & 0.29 & 18 & $\begin{array}{c}\mathrm{H}, 4-\mathrm{NH}_{2}, 2-\mathrm{Br}, 3-\mathrm{Br}, 4-\mathrm{Br}, 2-\mathrm{Cl} \text {, } \\
\text { 3-Cl, 4-Cl, 4-CN, 2-F, 3-F, 4-F, } \\
\text { 4- } \mathrm{OC}_{2} \mathrm{H}_{5}, 4-\mathrm{COOC}_{2} \mathrm{H}_{5}, 4-\mathrm{CH}_{3}, \\
\text { 4- } \mathrm{CH}_{3}, 4-\mathrm{NO}_{2}, 4-\mathrm{CF}_{3}\end{array}$ \\
\hline & $\sigma_{\mathrm{R}}$ & 0.836 & 66.748 & 0.535 & 0.34 & 18 & $\begin{array}{c}\mathrm{H}, 4-\mathrm{NH}_{2}, 2-\mathrm{Br}, 3-\mathrm{Br}, 4-\mathrm{Br}, 2-\mathrm{Cl} \text {, } \\
\text { 3-Cl, 4-Cl, 4-CN, 2-F, 3-F, 4-F, } \\
\text { 4- } \mathrm{OC}_{2} \mathrm{H}_{5}, 4-\mathrm{COOC}_{2} \mathrm{H}_{5}, 4-\mathrm{OCH}_{3} \text {, } \\
\text { 4- } \mathrm{CH}_{3}, 4-\mathrm{NO}_{2}, 4-\mathrm{CF}_{3}\end{array}$ \\
\hline & $\mathrm{F}$ & 0.849 & 66.332 & 0.758 & 0.31 & 18 & $\begin{array}{c}\mathrm{H}, 4-\mathrm{NH}_{2}, 2-\mathrm{Br}, 3-\mathrm{Br}, 4-\mathrm{Br}, 2-\mathrm{Cl}, \\
\text { 3-Cl, 4-Cl, 4-CN, 2-F, 3-F, 4-F, } \\
\text { 4- } \mathrm{OC}_{2} \mathrm{H}_{5}, 4-\mathrm{COOC}_{2} \mathrm{H}_{5}, 4-\mathrm{OCH}_{3}, \\
\text { 4- } \mathrm{CH}_{3}, 4-\mathrm{NO}_{2}, 4-\mathrm{CF}_{3}\end{array}$ \\
\hline & $\mathrm{R}$ & 0.836 & 66.756 & 0.461 & 0.33 & 18 & $\begin{array}{c}\mathrm{H}, 4-\mathrm{NH}_{2}, 2-\mathrm{Br}, 3-\mathrm{Br}, 4-\mathrm{Br}, 2-\mathrm{Cl} \text {, } \\
\text { 3-Cl, 4-Cl, 4-CN, 2-F, 3-F, 4-F, } \\
\text { 4-OC } \mathrm{OC}_{2} \mathrm{H}_{5}, 4-\mathrm{COOC}_{2} \mathrm{H}_{5}, 4-\mathrm{OCH}_{3}, \\
\text { 4- } \mathrm{CH}_{3}, 4-\mathrm{NO}_{2}, 4-\mathrm{CF}_{3}\end{array}$ \\
\hline
\end{tabular}

$\mathrm{r}=$ correlation coefficient, $\mathrm{I}=$ intercept, $\rho=$ slope, $\mathrm{s}=$ standard deviation, $\mathrm{n}=$ number of correlated derivatives

This is implies that there is a normal-substituent effects operates in all correlation. The correlation of $\delta \mathrm{Hb}$ chemical shifts (ppm) values correlated satisfactorily with Hammett $\sigma, \sigma^{+}$ substituent constants and $\mathrm{F}$ parameters individually except $\mathrm{NH}_{2}, 4-\mathrm{COOC}_{2} \mathrm{H}_{5}, 4-\mathrm{NO}_{2}, 4-\mathrm{CN}$ and $4-\mathrm{OC}_{2} \mathrm{H}_{5}$ substituents.

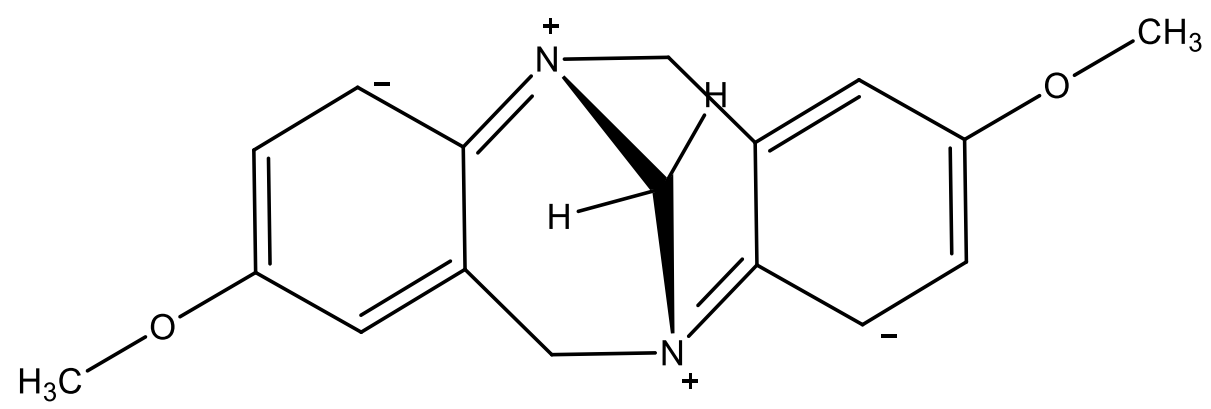

Fig. 2. The resonance - conjugative structure. 
If these substituents were included in the analysis, the result will be poor correlation. The remaining Hammett substituent constants and resonance parameters were fails in correlation. This is due to the inability of inductive and resonance effects of substituents on the methylene of all bases by opposing the lone pair of nitrogen atom and associated with the resonance conjugative structure shown in Fig. 2. The ratio of $\rho \mathrm{Ha}$ and $\rho \mathrm{Hb}$ is greater than 1 for $\sigma$ and $\sigma^{+}$. This implies that the transmittance of substituents effects on the methylene protons is higher extent for Ha chemical shifts(ppm) than $\mathrm{Hb}$.

In single parameter regression analysis, some of the correlations of $\delta \mathrm{Ha}$ and $\mathrm{Hb}$ chemical shifts (ppm) of Tröger's bases were fails with some Hammett substituent constants, $\mathrm{F}$ and $\mathrm{R}$ values. While seeking the multi linear correlation, these frequencies correlated satisfactorily with Inductive, Resonance and Swain-Lupton's[44] constants collectively and the correlated equations are given in 2-5.

$$
\begin{aligned}
& \delta \mathrm{Ha}^{(\mathrm{ppm})}=3.960( \pm 0.137)+0.717( \pm 0.295) \sigma_{\mathrm{I}}-0.334( \pm 0.238) \sigma_{\mathrm{R}} \\
& (R=0.957, \mathrm{n}=19, \mathrm{P}>95 \%) \\
& \delta \mathrm{Ha}^{(\mathrm{ppm})}=4.022( \pm 0.122)-0.627( \pm 0.249) \mathrm{F}-0.119( \pm 0.020) \mathrm{R} \\
& (R=0.957, \mathrm{n}=19, \mathrm{P}>95 \%) \\
& \delta \mathrm{Hb}^{(\mathrm{ppm})}=4.016( \pm 0.130)+0.679( \pm 0.280) \sigma_{\mathrm{I}}-0.357( \pm 0.226) \sigma_{\mathrm{R}} \\
& (R=0.958, \mathrm{n}=19, \mathrm{P}>95 \%) \\
& \delta \mathrm{Hb}^{(\mathrm{ppm})}=4.071( \pm 0.111)-0.225( \pm 0.228) \mathrm{F}-0.577( \pm 0.235) \mathrm{R} \\
& (R=0.959, \mathrm{n}=19, \mathrm{P}>95 \%)
\end{aligned}
$$

\section{2. ${ }^{13}$ C NMR Spectral study}

The Chemical shifts ( $\delta$, ppm) of methylene carbons of synthesised Tröger's bases were presented in Table 1. These chemical shifts $(\delta, \mathrm{ppm})$ have been correlated with Hammett substituent constants, $\mathrm{F}$ and $\mathrm{R}$ parameters using single and multi-linear regression analysis [32,34-44]. The results of statistical analysis are presented in Table 2. From the table 2, the methylene carbon chemical shifts $\left(\delta\right.$, ppm) of Tröger's bases with Hammett $\sigma, \sigma^{+}$and $\sigma_{I}$ constants were correlated satisfactorily. The remaining substituent constants, $\mathrm{F}$ and $\mathrm{R}$ parameters were failed in correlation. This is due to the reason stated earlier and associated with the resonance conjugative structure shown in Fig. 2. All correlation gave positive $\rho$ values. This means that there is a normal substituent effect operates in all systems.

The correlations of methylene carbon chemical shifts ( $\mathrm{ppm}$ ) of Tröger's bases were fails with some Hammett substituent constants, $\mathrm{F}$ and $\mathrm{R}$ values in single parameter regression analysis. When seeking the multi linear regression, it is worthwhile that these frequencies correlated satisfactorily with Inductive, Resonance and Swain-Lupton's[44] constants collectively and the correlated equations are given in 6 and 7 .

$$
\begin{aligned}
& \delta \mathrm{CH}_{2}{ }^{(\mathrm{ppm})}=66.325( \pm 0.600)+1.073( \pm 0.344) \sigma_{\mathrm{I}}+0.492( \pm 0.275) \sigma_{\mathrm{R}} \\
&(R=0.968, \mathrm{n}=19, \mathrm{P}>95 \%) \\
& \delta \mathrm{CH}_{2}{ }^{(\mathrm{ppm})}=66.432( \pm 0.142)+0.863( \pm 0.295) \mathrm{F}+0.574( \pm 0.243) \mathrm{R} \\
&(R=0.967, \mathrm{n}=19, \mathrm{P}>95 \%)
\end{aligned}
$$




\section{CONCLUSIONS}

A series of Tröger's bases have been synthesized by electrophilic substitution reaction of substituted anilines and paraformaldehyde in presence Lewis acid catalyst anhydrous $\mathrm{AlCl}_{3}$. The purities of these Tröger's bases were checked by their physical constants and spectroscopic data published earlier in the literature. The NMR spectroscopic frequencies $(\delta$, $\mathrm{ppm})$ of methylene protons and carbons were assigned. The assigned chemical shifts $(\delta, \mathrm{ppm})$ of methylene protons and carbons have been correlated with Hammett substituent constants, $\mathrm{F}$ and $\mathrm{R}$ parameters using single and multi-regression analyses. From the results of statistical analyses, the effects of substituents on methylene protons and carbon were discussed.

\section{ACKNOWLEDGEMENTS}

The author (Dr. G. T.) thanks (i). The UGC-Chemistry Network Resource Centre, School of Chemistry, Central University, Hyderabad - 500 040, for providing Summer Visiting Fellowship-June 2008, under the guidance of Prof. Dr. Mariappan Periasamy for initiating this Tröger's bases synthesizing work.

\section{References}

[1]. Bag G. G., Kiedrowski G., Tertrahdron Lett. 40 (1999) 1289-1290.

[2]. Prelog V., Wieland P., Helv. Chim. Acta. 27 (1944) 1127-1134.

[3]. Tálas E., Margitfalvi J., Machytka D., Czugler M., Tetrahedron Assymmetry 9 (1998) 4151-4156.

[4]. Carree F., Pardo C., Galy J-P., Boyer G., Robin M., Elguero G., Arkivoc.1 (2003) 1-8.

[5]. Brigita C., Liepinsh E., Vigante B., Sobolevs A., Ozols J., Duburs G., Tetrahedron Lett. 42 (2001) 4239-4241.

[6]. Depres N. R., McNitt K. A., Petersen M. E., Brown R. G., Lewis D. E., Tetrahedron Lett. 46 (2005) 2149-2153.

[7]. Hansson A., Norrby P-O., Warnmark K., Tetrahedron Lett. 39 (1998) 4565-4568.

[8]. Kim K., Choe J-I., Bull. Korean Chem. Soc. 27(11) (2006) 1737-1740.

[9]. Wu H., Chen, X-C. Wan Y., Ye L., Xin H., Xu H., Yue C., Pang L., Ma R., Shi D. Tetrahedron Lett. 50 (2009) 1062-1065.

[10]. Faroughi M., Jenssen P., Try A. C., Arkivoc. 2(2009) 269-280.

[11]. Abonia R., Rengifo E., Quiroga J., Insuasty B., Sanchez A., Cobo J., Low J., Nogueras, M., Tetrahedron Lett. 43(2002) 5617-5620.

[12]. Valík M., Dolensky B., Petříčková H., Vašek P., Král V., Tetrahedron Lett. 44(2003) 2083-2086.

[13]. Abella C. A. M., Rodembusch F. S., Stefani V., Tetrahedron Lett. 45(2004) 56015604

[14]. Sathishkumar S., Periasamy M., Tetrahedron: Assym. 17 (2006) 1116-1119.

[15]. Sathishkumar S., Periasamy M., Tetrahedron: Assym. 20 (2009) 2257-2262.

[16] Mahon A. B., Craig D. C., Try A. C., Arkivoc. 12(2008) 148-163. 
[17]. Vardelle E., Agnes M-M., Jouannetaud M-P., Jacquesy J-C., Marrot J., Tetrahedron Lett. 50 (2009) 1093-1096.

[18]. Didier D., Sergeyev S., Tetrahedron 63 (2007) 3864-3869.

[19]. Try A. C., Painter L., Harding M M., Tetrahedron Lett. 39 (1998) 9809-9812.

[20]. Adbo K., Andersson H. S., Ankarloo J., Karlsson J. G., Norell M. C., Olofsson L., Svenson J., Ortegren U., Nicholls I. A. Bioorg. Chem. 27 (1999) 363-371.

[21]. Bresson C., Luhmer M., Demeunynck M., Mesmaeker A. K-D., Pierard F., Tetrahedron Lett. 45(2004) 2863-2866.

[22]. Kiehne U., Weilandt T. Y., Lutzen A., Org. Lett. 9(7) (2007) 1283-1286.

[23]. Hansson A., Jensen J., Wendt O. F., Warnmark K., Eur. J. Org. Chem. 7 (2003) 31793188 .

[24]. Pardo C., Alkorta I., Elguero J., Tetrahedron: Assym. 17(2006)191-198.

[25]. Mas T., Pardo C., Elguero J., Mandeleev Commun. 14(6) (2004) 235-237.

[26]. Goswami S., Ghosh K., Dasgupta S., J. Org. Chem. 65 (2000) 1907-1914.

[27]. Valik M., Malina J., Foltynova J., Tkadlecova M., Urbanova M., Brabec V., Kral, V. Tetrahedron 62 (2006) 8591-8600.

[28]. Sergeyev S., Stas S., Remacle A., Velde C. M. L. V., Dolensky B., Havlik M., Kral V., Cejka J., Tetrahedron: Assym. 20 (2009) 1918-1923.

[29]. Mihlbachler K., Kaczmarski K., Morgenstern A. S., Guiochon G., J. Chromatograph. 955A (2002) 35-52.

[30]. Putnam J., Guiochon G., J. Chromatograph. 1216A (2009) 8488-8495.

[31]. Classens N., Perard F., Bresson C., Moucheron C., Mesmaeker A. K-D., J. Inorg. Biochem. 101 (2007) 987-996.

[32]. Arulkumaran R., Vijayakumar S., Sundararajan R., Sakthinathan S. P., Kamalakkannan D., Suresh R., Ranganathan K., Vanangamudi G., Thirunarayanan G., International Letters of Chemistry, Physics and Astronomy 4 (2012) 17-38.

[33]. Thirunarayanan G., Arab. J. Chem. (2012). DOI. http://dx.doi.org/10.1016/ j.arabjc.2012.10.025.

[34]. Ranganathan K., Suresh R., Kamalakkannan D., Arulkumaran R., Sundararajan R., Sakthinathan S. P., Vijayakumar S., Vanangamudi G., Thirumurthy K., Mayavel P., Thirunarayanan G., International Letters of Chemistry, Physics and Astronomy 4 (2012) 66-75.

[35]. Arulkumaran R., Vijayakumar S., Sundararajan R., Sakthinathan S. P., Kamalakkannan D., Suresh R., Ranganathan K., Rajakumar P. R., Vanangamudi G., Thirunarayanan G., International Letters of Chemistry, Physics and Astronomy 5 (2013) 21-38.

[36]. Sakthinathan S. P., Suresh R., Mala V., Sathiyamoorthi K., Kamalakkannan D., Ranganathan K., Arulkumaran R., Vijayakumar S., Sundararajan R., Vanangamudi G., Thirunarayanan G., International Letters of Chemistry, Physics and Astronomy 6 (2013) 77-90. 
[37]. Sathiyamoorthi K., Mala V., Suresh R., Sakthinathan S. P., Kamalakkannan D., Ranganathan K., Arulkumaran R., Sundararajan R., Vijayakumar S., Vanangamudi G., Thirunarayanan G., International Letters of Chemistry, Physics and Astronomy 7(2) (2013) 102-119.

[38]. Sekar K. G., Thirunarayanan G., International Letters of Chemistry, Physics and Astronomy 8(2) (2013)160-174.

[39]. Sekar K. G., Thirunarayanan G., International Letters of Chemistry, Physics and Astronomy 8(3) (2013) 249-258,

[40]. Thirunarayanan G., International Letters of Chemistry, Physics and Astronomy 9(2) (2013) 152-161.

[41]. Vijayakumar S., Arulkumaran R., Sundararajan R., Sakthinathan S. P., Suresh, R., Kamalakkannan D., Ranganathan K., Sathiyamoorthy K., Mala V., Vanangamudi G., Thirunarayanan G., International Letters of Chemistry, Physics and Astronomy 9(1) (2013) 68-86.

[42]. Thirunarayanan G., Sekar K. G., International Letters of Chemistry, Physics and Astronomy 10(1) (2013) 18-34.

[43]. Thirunaryanan G., Ravi K., International Letters of Chemistry, Physics and Astronomy 14 (2013) 44-57.

[44]. Swain C. G., Lupton Jr., E. C. J. Am. Chem. Soc. 90 (1968) 4328-4337. 